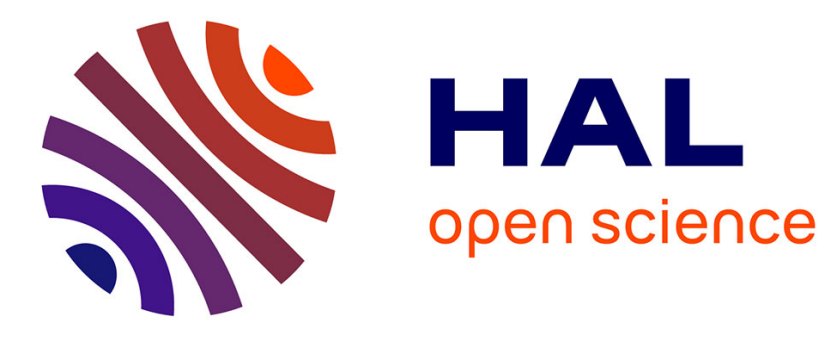

\title{
Stability and Stabilisation Through Envelopes for Retarded and Neutral Time-Delay Systems
}

Caetano Cardeliquio, André Fioravanti, Catherine Bonnet, Silviu-Iulian Niculescu

\section{- To cite this version:}

Caetano Cardeliquio, André Fioravanti, Catherine Bonnet, Silviu-Iulian Niculescu. Stability and Stabilisation Through Envelopes for Retarded and Neutral Time-Delay Systems. IEEE Transactions on Automatic Control, inPress, 65 (4), pp.1640-1646. 10.1109/TAC.2019.2929092 . hal-02350927

\section{HAL Id: hal-02350927 \\ https://hal-centralesupelec.archives-ouvertes.fr/hal-02350927}

Submitted on 12 Jan 2022

HAL is a multi-disciplinary open access archive for the deposit and dissemination of scientific research documents, whether they are published or not. The documents may come from teaching and research institutions in France or abroad, or from public or private research centers.
L'archive ouverte pluridisciplinaire HAL, est destinée au dépôt et à la diffusion de documents scientifiques de niveau recherche, publiés ou non, émanant des établissements d'enseignement et de recherche français ou étrangers, des laboratoires publics ou privés. 


\title{
Stability and Stabilisation Through Envelopes for Retarded and Neutral Time-Delay Systems
}

\author{
Caetano B. Cardeliquio, André R. Fioravanti, Catherine Bonnet and Silviu-Iulian Niculescu.
}

\begin{abstract}
This paper deals with a new approach to develop an envelope that engulfs all poles of a time-delay system. Through LMIs we are able to determine envelopes for retarded and neutral time-delay systems. The envelopes proposed are not only tighter than the ones in the literature but, with our procedure, they can also be applied to verify the stability of the system and design state-feedback controllers which cope with design requirements regarding $\alpha$ - stability.
\end{abstract}

Index Terms-Neutral-type, Retarded-type, Stabilisation, Stability, State-Feedback, Time-delay systems

\section{INTRODUCTION}

Time-delay systems have instigated an increasingly interest from the control community. The main reason is that they are intrinsically coupled with almost every dynamical system. This is due to delays originated from transport, processing time, sampling, propagation time on networked systems, among others. Sometimes it is not possible to neglect those delays because they may cause bad performance or even instability. Stability for time-delay systems was discussed, among others, in [1], [2] and [3].

For the stabilisation through state feedback, delayindependent controllers can be devised using Riccati equations [4], [5], whereas the delay-dependent case was designed by means of Lyapunov-Krasoviskii functionals in [6]-[8]. A controller design approach through a finite LTI comparison system was developed in [9]. The use of an envelope that ensures that all poles are contained inside it was discussed in [1]. Different types of envelopes were also discussed in [10] and [11]. In any case, to the best of our knowledge, no methods utilising envelopes were developed to test stability nor to design controllers. In fact, in general, the envelope extends to the right half-plane and therefore, it only provides a region where the poles are allowed to be without any guarantee about the stability of the system. In this work we provide a different analysis for the use of envelopes. Instead of using a singular value approach, such as in [1], our method is based on Linear Matrix Inequalities (LMIs). We are able to provide a new procedure to test stability for both retarded and neutral time-delay systems. Furthermore, it allows to cope with some

This work was supported by grants from Smart ${ }^{2}$ Erasmus Mundus, Fundao de Amparo Pesquisa do Estado de So Paulo (FAPESP - 2018/049051) and Conselho Nacional de Desenvolvimento Científico e Tecnológico (CNPq/Brazil - 305600/2017-6)

Caetano B. Cardeliquio, Catherine Bonnet and Silviu-Iulian Niculescu are with Inria, L2S-CNRS, Supélec, 3 rue Joliot Curie, 91192, Gif-sur-Yvette, France (e-mail: caetano.cardeliquio, catherine.bonnet, silviu.niculescu@12s.centralesupelec.fr).

André R. Fioravanti is with School of Mechanical Engineering, University of Campinas, Brazil (e-mail: fioravanti@fem.unicamp.br). project requirements designing a state-feedback controller that guarantees $\alpha$-stability.

Notation. Matrices are denoted by capital letters, whilst small letters represent scalars and vectors. For real matrices or vectors the symbol $\left({ }^{\prime}\right)$ indicates transpose and for complex matrices or vectors the symbol $(*)$ denotes conjugate transpose. The determinant of a matrix $A$ is indicated by $\operatorname{det}(A)$. The sets of real, integer and natural numbers including zero are denoted by $\mathbb{R}, \mathbb{Z}$ and $\mathbb{N}$, respectively. Floor is defined as $\lfloor x\rfloor=\max \{m \in \mathbb{Z} \mid m \leq x\}, x \in \mathbb{R} . \Re($.$) is the real part of a$ complex number. A left eigenvector is defined as a row vector $x_{L}$ satisfying $x_{L} A=\lambda_{L} x_{L}$, where $\lambda_{L}$ is a left eigenvalue of the matrix $A$. For partitioned matrices the symbol $\bullet$ represents each one of its Hermitian blocks. The induced $p$-norm of a matrix $A$ is given by $\|A\|_{p}, A \in \mathbb{C}^{n \times m}$. Finally, $X>0$ $(X \geq 0)$ denotes that the symmetric matrix $X$ is positive definite (positive semi-definite).

\section{RETARDED SYSTEMS}

Consider the retarded linear time-delay system with $N$ delays, whose minimal realisation is given by

$$
\dot{x}(t)=\sum_{i=0}^{N} A_{i} x\left(t-\tau_{i}\right),
$$

where $x(t) \in \mathbb{R}^{n}$ is the state variable, $0=\tau_{0}<\tau_{1}<\cdots<\tau_{N}$ are the delays and $A_{i} \in \mathbb{R}^{n \times n}$ for all $i \in\{0, \ldots, N\}$. This system is exponentially stable if and only if all roots of its characteristic equation

$$
\operatorname{det}\left(s I-\sum_{i=0}^{N} A_{i} e^{-s \tau_{i}}\right)=0
$$

are in the open left half-plane [12].

The following Proposition introduces an envelope that engulfs all of its poles.

Proposition 1: Let $\lambda$ be any real number. If there exist matrices $T=T^{\prime}>0, Q_{i}=Q_{i}^{\prime}>0$, for all $i \in\{0, \ldots, N\}$ and a scalar $\mu$ that satisfy

$$
\mu T \geq \sum_{i=0}^{N} A_{i} Q_{i} A_{i}^{\prime} e^{-2 \lambda \tau_{i}}
$$

and

$$
\left[\begin{array}{cccc}
T & T & \ldots & T \\
\bullet & Q_{0} & & 0 \\
\bullet & \bullet & \ddots & 0 \\
\bullet & \bullet & \bullet & Q_{N}
\end{array}\right] \geq 0
$$


then any characteristic root $s_{0}$ of equation (2) such that $s_{0}=$ $\lambda+\jmath \omega$ verifies

$$
\left|s_{0}\right| \leq \sqrt{\mu}
$$

Proof: The following inequality is always true, which is easily verifiable applying Schur's complement

$$
\left[\begin{array}{cc}
A_{i} Q_{i} A_{i}^{\prime} e^{-2 \lambda \tau_{i}} & \bullet \\
A_{i}^{\prime} e^{-(\lambda-\jmath \omega) \tau_{i}} & Q_{i}^{-1}
\end{array}\right] \geq 0 .
$$

Adding them for all $i \in\{0, \ldots, N\}$ leads to

$$
\left[\begin{array}{cc}
\sum_{i=0}^{N} A_{i} Q_{i} A_{i}^{\prime} e^{-2 \lambda \tau_{i}} & \bullet \\
\sum_{i=0}^{N} A_{i}^{\prime} e^{-(\lambda-\jmath \omega) \tau_{i}} & \sum_{i=0}^{N} Q_{i}^{-1}
\end{array}\right] \geq 0,
$$

where we can apply Schur's complement and utilise (3) to get

$$
\mu T \geq \Sigma\left(\sum_{i=0}^{N} Q_{i}^{-1}\right)^{-1} \Sigma^{*},
$$

where $\Sigma \triangleq \sum_{i=0}^{N} A_{i} e^{-(\lambda+\jmath \omega) \tau_{i}}$

Notice that from (4)

$$
T \geq \sum_{i=0}^{N} T Q_{i}^{-1} T .
$$

Now, multiplying (9), through the left and through the right, by $T^{-1}$ and taking the inverse on both sides of the inequality, we get

$$
T \leq\left(\sum_{i=0}^{N} Q_{i}^{-1}\right)^{-1} .
$$

Then, using this result in (8), it implies that

$$
\mu T \geq \Sigma T \Sigma^{*}
$$

Finally, let $s_{0}=\lambda+j \omega$ be an eigenvalue of $\Sigma$ associated with a right-eigenvector $v$. It is well known, [13] and [14], that left and right eigenvalues are equal. Hence, $s_{0}$ is also an eigenvalue of $\Sigma$ associated with a left-eigenvector $x_{L}$, with dimension $1 \times n$. In this case, we can multiply inequality (11) to the left by $x_{L}$ and to the right by its conjugated transpose, $x_{L}^{*}$, obtaining

$$
\mu x_{L} T x_{L}^{*} \geq x_{L} \Sigma T \Sigma^{*} x_{L}^{*}
$$

and since $x_{L} \neq 0$ and $T>0$,

$$
\mu \geq(\lambda+\jmath \omega)(\lambda-\jmath \omega)
$$

leading to

$$
\left|s_{0}\right| \leq \sqrt{\mu}
$$

which concludes the proof.

This result produces a better envelope than previous works such as [1]. This will be proved in the next Lemma and evidenced in Example 1.
Let us analyse the envelope obtained from the eigenvalue approach to hereafter introduce a Lemma that compares it with the LMI approach. The envelope in [1] is given by

$$
\left|s_{0}\right| \leq \sum_{i=0}^{N}\left\|A_{i}\right\|_{2} e^{-\lambda \tau_{i}},
$$

which is equivalent, see [15], to $\left|s_{0}\right| \leq \nu$, where $\nu$ is the optimal solution of the following optimisation problem

$$
\begin{array}{cl}
\min _{\nu, \nu_{i}} & \nu, \\
\text { subject to } & \nu \geq \sum_{i=0}^{N} \nu_{i}, \\
& \nu_{i}^{2} I \geq A_{i}^{\prime} A_{i} e^{-2 \lambda \tau_{i}},
\end{array}
$$

for all $i \in\{0, \ldots, N\}$. and $\nu_{i} \geq 0$, for all $i \in\{0, \ldots, N\}$.

The next Lemma shows that the envelope in [1] is a particular case of the class of envelopes defined by Proposition 1

Lemma 1: Let $s_{0}=\lambda+\jmath \omega$ be a characteristic solution of equation (2) and let $\nu$ and $\nu_{i}$ be the optimal solution of the optimisation problem (16). Then (3) and (4) are both satisfied with the particular choice of $T=\nu^{-1} I, Q_{i}=\nu_{i}^{-1} I$ and $\mu=\nu^{2}$.

Proof: From Schur Complement, (4) is equivalent to

$$
T \geq \sum_{i=0}^{N} T Q_{i}^{-1} T \text {. }
$$

It is easy to see that (17) is satisfied whenever $T=\nu^{-1} I$ and $Q_{i}=\nu_{i}^{-1} I$.

Applying the same substitutions on (3), we get

$$
\mu \nu^{-1} I \geq \sum_{i=0}^{N} A_{i} \nu_{i}^{-1} A_{i}^{\prime} e^{-2 \lambda \tau_{i}}
$$

and remembering that $\mu=\nu^{2}$, we have

$$
\sum_{i=0}^{N} \nu_{i} I \geq \sum_{i=0}^{N} A_{i} \nu_{i}^{-1} A_{i}^{\prime} e^{-2 \lambda \tau_{i}},
$$

which satisfies the conditions in (16).

Therefore, the envelope in [1] is a particular case of Proposition 1 for specific choices of $T, Q_{i}$ and $\mu$. Having flexibility on those three variables allows the proposed new envelope to provide tighter (or at least equal) bounds than the envelope aforementioned.

\section{A. Implementation}

First of all, let us introduce the definition of closeness of an envelope. Let $\mu$ and $\lambda$ be defined by Proposition 1 and let $\lambda \in\left[\lambda_{\min }, \lambda_{\max }\right]$. If there is a point $\lambda^{*}$ in this interval such that $\mu=\left(\lambda^{*}\right)^{2}$, we define $\lambda^{*}+\varepsilon$, with $\varepsilon>0$ arbitrarily small, as the closure point of the envelope. This means that the envelope lies completely on the left side of the vertical line $\Re(s)=\lambda^{*}+\varepsilon$. Furthermore, we say that the envelope is closed whenever $\mu<\lambda^{2}$. 
The choice of $\lambda_{\min }$ is completely free. In [10], a simple bound for the rightmost root of (2) was given, which can easily be generalised to $N$ delays:

$$
\Re(s) \leq \mu\left(A_{0}\right)+\sum_{i=1}^{N}\left\|A_{i}\right\|=\ell,
$$

where $\mu($.$) is a matrix measure, see [10] and [16]. We suggest$ to take $\lambda_{\max }=2|\ell|$.

The following propositions illustrate, respectively, how to depict the envelope and how one can use the envelope to analyse the stability of a time delay system. Also, it shows the behaviour of the envelope as a function of $\lambda$.

Proposition 2: Let $\lambda \in\left[\lambda_{\min }, \lambda_{\max }\right]$ and let $\mu$ be given by (3). If $\mu \geq \lambda^{2}$ then the envelope on the complex plane is defined by the set of points $(\lambda, \omega)$ where $\omega= \pm \sqrt{\mu-\lambda^{2}}$. If for a particular $\lambda^{*}, \mu^{*}<\left(\lambda^{*}\right)^{2}$ then the envelope is closed for every $\lambda>\lambda^{*}$.

Proof: From equation (14) we have that $\lambda^{2}+\omega^{2} \leq \mu$ which directly implies that $\omega= \pm \sqrt{\mu-\lambda^{2}}$, for $\mu \geq \lambda^{2}$. Obviously, $(\lambda, \omega)$ belongs to the envelope. Now, suppose that for a certain $\lambda^{*}$, we have $\mu^{*}<\left(\lambda^{*}\right)^{2}$. As $A_{i} Q_{i} A_{i}^{\prime} \geq 0$ and $e^{-2 \lambda \tau_{i}}$ is nonincreasing, we have that $\mu<\mu^{*}$ for every $\lambda>\lambda^{*}$ which means, by definition, that the envelope is closed.

Proposition 3: Let $\lambda_{0} \in \mathbb{R}$ and $\mu=\lambda_{0}^{2}-\varepsilon$, for some $\varepsilon>0$. If there exist $T, Q_{i}>0$, for all $i \in\{0, \ldots, N\}$ such that (3) and (4) are both satisfied, then the envelope lies entirely on the left side of the vertical axis crossing $\lambda_{0}$.

Proof: From (14) we have that if $\lambda+\jmath \omega$ is a root of the system, then

$$
|\lambda+\jmath \omega| \leq \sqrt{\lambda_{0}^{2}-\varepsilon},
$$

which can be rewritten as

$$
\lambda^{2}+\omega^{2} \leq \lambda_{0}^{2}-\varepsilon .
$$

Notice that this expression is never going to be satisfied with $\lambda \geq \lambda_{0}$, which implies that it cannot exist parts of the envelope to the right side of the vertical axis passing through $\lambda_{0}$.

The computational procedure to obtain the envelope is summarized in the Algorithm 1. The minimization of $\mu$, for the retarded case, is achieved through the traditional generalised eigenvalue minimisation under LMI constraints, [17]. For the neutral case, which will be discussed further ahead, the minimisation of $\mu$ is done through a linear search, i.e., we choose a $\mu_{0}$ using the generalised eigenvalue problem (gevp) and proceed through a linear search on $\mu$ checking on each step the feasibility of the LMIs. Since LMIs are convex and gevp is quasi-convex, there is no need for initial values for convergence to the optimal solution.

In spite of the fact that this envelope is tighter than [1], for $\lambda=0$, it follows from (3) that $\mu \geq 0$, and therefore, the envelope is never closed on the left half-plane, which implies that stability cannot be assessed with the envelope in this present form. To circumvent this, we propose a change of coordinates through the new variable $s=z-d$, with $d>0$

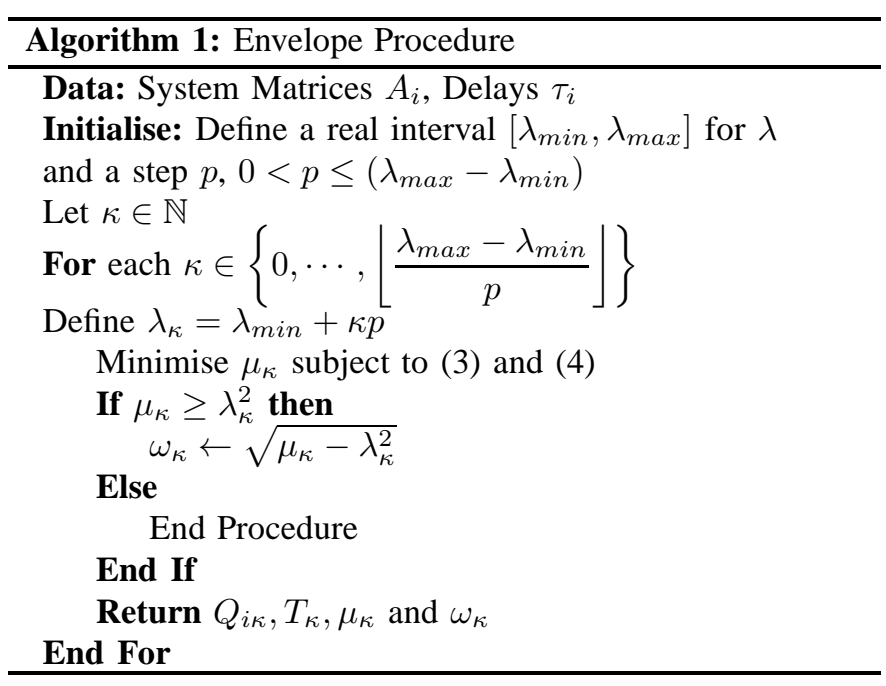

and hereafter calculate the envelope for $z$. With this change of variables, (2) becomes

$$
\operatorname{det}\left(z I-\left(A_{0}+d I\right)-\sum_{i=1}^{N} A_{i} e^{-z \tau_{i}} e^{d \tau_{i}}\right)=0,
$$

allowing us to work with an equivalent problem on the new parameters

$$
\begin{aligned}
& \tilde{A}_{0}=A_{0}+d I, \\
& \tilde{A}_{i}=A_{i} e^{d \tau_{i}}, \text { for all } i \in\{1, \ldots, N\} .
\end{aligned}
$$

On the $z$-plan the envelope will remain open for $\lambda=0$, however, if it is closed before $z=d$, it will be closed before the origin on the $s$-plan, guaranteeing stability for the original system.

Example 1: Consider the following system matrices

$$
\left[A_{0} \mid A_{1}\right]=\left[\begin{array}{rr|rr}
0 & 1 & 0 & 0.5413 \\
-2 & -3 & -1.0827 & -1.6240
\end{array}\right] \text {. }
$$

Applying Algorithm 1, for $\tau_{1}=1$, to this system, we calculate the envelope and compare the result with reference [1]. Figure 1 shows this comparison and it also illustrates the behaviour of the envelope for different values of $d$. An interesting remark is that for $d=3$ we achieved a tighter envelope closer to the poles and we can also see that the point where the envelope ends is on the left side of the plane. This allows us to use the envelope as a stability criteria as will be seen in the stabilisation section. All system poles here and throughout this work were calculated via QPmR, [18] and [19].

\section{B. Stability}

There are two types of stability that can be analysed and obtained using the envelope.

1) Delay independent stability: Proposition 3 shows that the existence of a solution for (3) and (4), for the modified system (24), with $\mu=d^{2}-\varepsilon$ and $\lambda=d$, for some $d>0$ and $\varepsilon>0$ implies that the original system (1) is stable. Note also that, for $\lambda=d$, after the change of variables (24), all terms of inequality (3) that have delays cancel each other. This implies that the criteria is delay independent. 


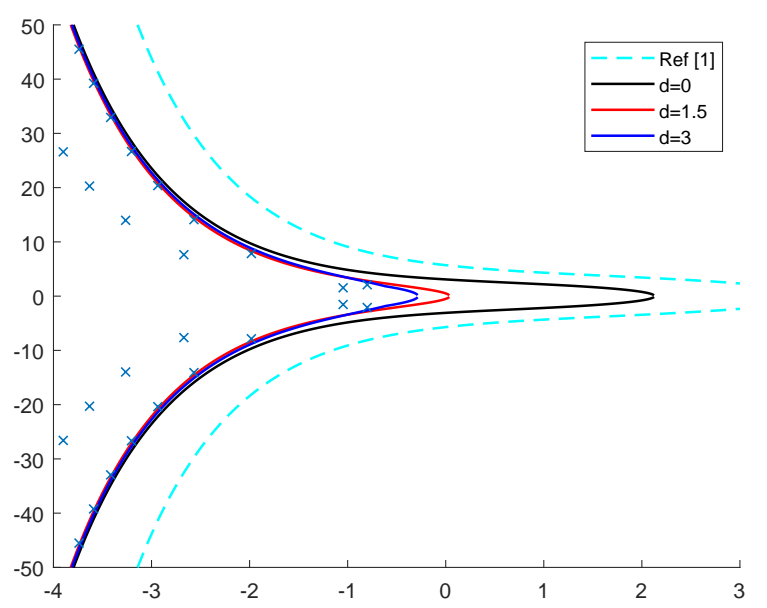

Fig. 1: Envelopes for different values of $d$ and from previous work in the literature

2) Delay dependent $\alpha$-stability: Is it possible to go one step further and design a controller that guarantees $\alpha$-stability. Making the change of variables $z=s+d$, with $d=d^{*}+\alpha$, $d^{*}>0, \alpha>0$, it implies that if an envelope lies completely before $d^{*}$ on the $z$-plane, then it will lies completely on the left side of the vertical line $\Re(s)=-\alpha$ on the $s$-plan.

For this case, with $\mu=\left(d^{*}\right)^{2}-\varepsilon$ and $\lambda=d^{*}$, (3) becomes

$$
\mu T \geq \tilde{A}_{0} Q_{0} \tilde{A}_{0}^{\prime}+\sum_{i=1}^{N} A_{i} Q_{i} A_{i}^{\prime} e^{2 \alpha \tau_{i}}
$$

Now the criteria is delay dependent. Furthermore, if 25 is satisfied for $\alpha=\alpha^{*}$ and $\tau_{i}=\tau_{i}^{*}$ for all $i \in\{1 \cdots N\}$ then it will remain $\alpha$-stable for all $\tau_{i} \leq \tau_{i}^{*}$.

\section{State-feedback for Retarded systems}

We now address the stabilisation problem. Consider the system

$$
\dot{x}(t)=\sum_{i=0}^{N} A_{i} x\left(t-\tau_{i}\right)+B u(t),
$$

which we want to be controlled by means of a state feedback control law

$$
u(t)=\sum_{i=0}^{N} K_{i} x\left(t-\tau_{i}\right) \in \mathbb{R}^{m},
$$

to be designed through LMIs. This controller copes with project requirements, i.e., $\alpha$-stability, and adds a certain degree of robustness to the closed-loop system. As will be shown the controller can be memoryless, i.e., $K_{i} \leftarrow 0, \forall i \in\{1, \ldots, N\}$ or can even use only some of the delayed states.

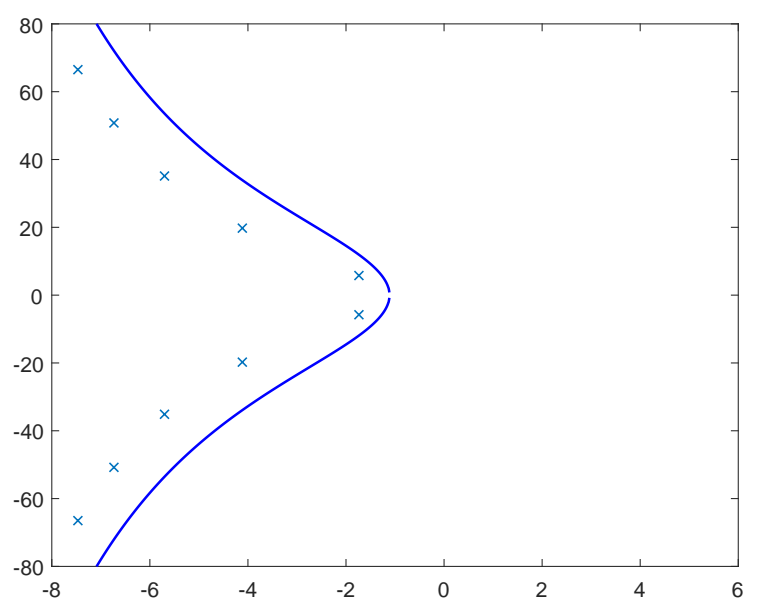

Fig. 2: $\alpha$-stability, $\alpha=1$

Theorem 1: Consider the time-delay system (26). If there exist matrices $T=T^{\prime}>0, Q_{i}=Q_{i}^{\prime}>0, Y_{i} \forall i \in\{0, \ldots, N\}$ and positive scalars $d$, $\varepsilon$, with $\mu=d^{2}-\varepsilon, \lambda=d$, such that

$$
\left[\begin{array}{cccc}
\mu T & \left(\tilde{A}_{0} Q_{0}+B_{0} Y_{0}\right) e^{-\lambda \tau_{0}} & \ldots & \left(\tilde{A}_{N} Q_{N}+B_{N} Y_{N}\right) e^{-\lambda \tau_{N}} \\
\bullet & Q_{0} & & 0 \\
\bullet & \bullet & \ddots & 0 \\
\bullet & \bullet & Q_{N}
\end{array}\right] \geq 0
$$

and (4) are all satisfied, where $\tilde{A}_{i}$ is given by (24) and $B_{i}=$ $B e^{d \tau_{i}}$ for all $i \in\{0, \ldots, N\}$, then the state-feedback control law (27), where the controller matrices are given by $K_{i}=$ $Y_{i} Q_{i}^{-1}$, stabilises the system.

Proof: Applying Schur's complement in (28) we get exactly (3) with $A_{i} \leftarrow \tilde{A}_{i}+B_{i} K_{i}$, which completes the proof.

Example 2: Taking the matrices $-A_{0}$ and $-A_{1}$, from Example 1, with $\tau=0.4$ and $B=\left[\begin{array}{ll}0 & 1\end{array}\right]^{\prime}$, the uncontrolled system is unstable with poles at 1.3194 and 2.4125 . Choosing $\alpha=1, d=31, \lambda=d-\alpha$ and applying Theorem 11 we achieve $\alpha$-stability as can be seen in Figure 2. The gains for the controller are

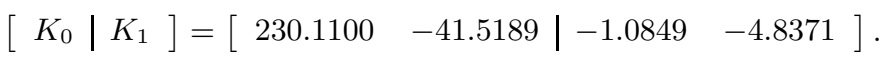

We can also impose, for example, $K_{1}=0$ and still achieve $\alpha$-stability. In that case $K_{0}=[298.9831-43.7406]$.

Using (20) for the change of variables, i.e., $d=\mu\left(A_{0}\right)+$ $\left\|A_{1}\right\|$, we get

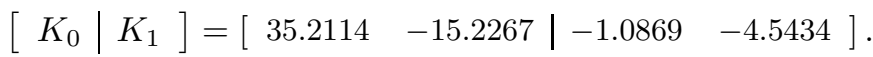

which has not only a smaller gain norm but also a tighter envelope as can be seen in the Figure 3 .

Remark 1: Results presented here are in some sense complementary to those obtained from Lyapunov-Krasoviskii functionals. In general, Lyapunov-Krasoviskii methods are able to cope with a larger class of systems, such as timevarying delays [26] and [27], as well as providing guaranteed performance metrics such as $\mathcal{H}_{\infty}$ [28]. On the other hand, results built on frequency methods are more restricted with respect to the class of systems they may be applied, but are 


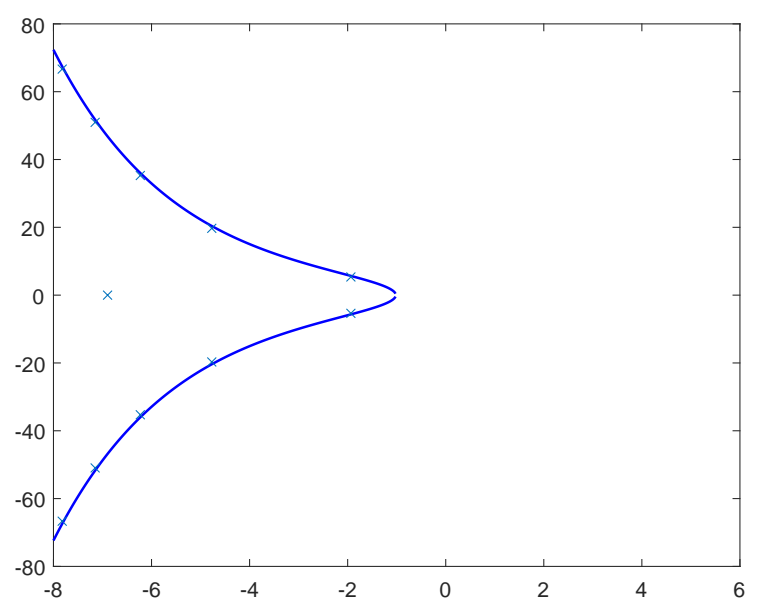

Fig. 3: $\alpha$-stability, $\alpha=1$

able to provide information on the position of poles, and therefore on performance metrics which are more directly related to them.

\section{Neutral Systems}

Our goal here is to develop the envelopes for neutral-type systems. Consider the neutral time-delay linear system with $N+1$ delays, whose minimal realisation is given by

$$
\dot{x}(t)=\sum_{i=0}^{N} A_{i} x\left(t-\tau_{i}\right)+H \dot{x}\left(t-\tau_{h}\right),
$$

where $x(t) \in \mathbb{R}^{n}$ is the state variable, $0=\tau_{0}<\tau_{1}<\cdots<\tau_{N}$ and $\tau_{h}$ are the delays, $A_{i} \in \mathbb{R}^{n \times n}$, for all $i \in\{0, \ldots, N\}$ and $H$ are real matrices. One sufficient condition for the exponential stability of this system is that all roots of the characteristic equation

$$
\operatorname{det}\left(s I-\sum_{i=0}^{N} A_{i} e^{-s \tau_{i}}-s H e^{-s \tau_{h}}\right)=0,
$$

are on the left side of a vertical line $\Re(s)=-\alpha$, with $\alpha>0$ [20]. The following Proposition generalises the envelope for neutral systems.

Proposition 4: Let $\lambda$ be any real number. If there exist matrices $T=T^{\prime}>0, Q_{i}=Q_{i}^{\prime}>0, \forall i \in\{0, \ldots, N\}$, $Q_{h}=Q_{h}^{\prime}>0$ and a scalar $\mu$ such that

$$
\mu T \geq \sum_{i=0}^{N} A_{i} Q_{i} A_{i}^{\prime} e^{-2 \lambda \tau_{i}}+\mu H Q_{h} H^{\prime} e^{-2 \lambda \tau_{h}}
$$

and

$$
\left[\begin{array}{ccccc}
T & T & \cdots & T & T \\
\bullet & Q_{0} & & 0 & 0 \\
\bullet & \bullet & \ddots & 0 & 0 \\
\bullet & \bullet & \bullet & Q_{N} & 0 \\
\bullet & \bullet & \bullet & \bullet & \frac{\mu}{\left|s_{0}\right|^{2}} Q_{h}
\end{array}\right]>0
$$

then any characteristic root $s_{0}$ of equation (30) such that $s_{0}=$ $\lambda+\jmath \omega$ verifies

$$
\left|s_{0}\right| \leq \sqrt{\mu}
$$

Proof: The following inequality is always true, which is easily verifiable applying Schur's complement

$$
\left[\begin{array}{cc}
H Q_{h} H^{\prime} e^{-2 \lambda \tau_{h}} & \bullet \\
s_{0}^{*} H^{\prime} e^{-(\lambda-\jmath \omega) \tau_{h}} & \left|s_{0}\right|^{2} Q_{h}^{-1}
\end{array}\right] \geq 0
$$

Multiplying both sides by $\operatorname{diag}\left(\sqrt{\mu}, \frac{1}{\sqrt{\mu}}\right)$ and then adding the result to (7) we get

$$
\left[\begin{array}{cc}
\sum_{i=0}^{N} A_{i} Q_{i} A_{i}^{\prime} e^{-2 \lambda \tau_{i}}+\mu H Q_{h} H^{\prime} e^{-2 \lambda \tau_{h}} & \bullet \\
\sum_{i=0}^{N} A_{i}^{\prime} e^{-(\lambda-\jmath \omega) \tau_{i}}+s_{0}^{*} H^{\prime} e^{-(\lambda-\jmath \omega) \tau_{h}} & \sum_{i=0}^{N} Q_{i}^{-1}+\frac{\left|s_{0}\right|^{2}}{\mu} Q_{h}^{-1}
\end{array}\right] \geq 0,
$$

where we can apply Schur's complement and utilise (31) to get

$$
\mu T \geq \Sigma\left(\sum_{i=0}^{N} Q_{i}^{-1}+\frac{\left|s_{0}\right|^{2}}{\mu} Q_{h}^{-1}\right)^{-1} \Sigma^{*},
$$

where $\Sigma \triangleq \sum_{i=0}^{N} A_{i} e^{-(\lambda+\jmath \omega) \tau_{i}}+s_{0} H e^{-(\lambda+\jmath \omega) \tau_{h}}$. Furthermore, from (32) we have that

$$
T>\sum_{i=0}^{N} T Q_{i}^{-1} T+\frac{\left|s_{0}\right|^{2}}{\mu} T Q_{h}^{-1} T,
$$

which implies

$$
T<\left(\sum_{i=0}^{N} Q_{i}^{-1}+\frac{\left|s_{0}\right|^{2}}{\mu} Q_{h}^{-1}\right)^{-1} .
$$

Therefore, using this result with (36), it implies that

$$
\mu T \geq \Sigma T \Sigma^{*}
$$

Proceeding in the same manner as the retarded case, multiplying the inequality 39] to the left by $x_{L}$ and to the right by $x_{L}^{*}$, where $x_{L}$ is the left-eigenvector of $\Sigma$ associated to the eigenvalue $s_{0}$, leads to

$$
\left|s_{0}\right| \leq \sqrt{\mu}
$$

One difficulty on applying this method lies on the necessity of knowing $\left|s_{0}\right|$ to implement the LMI (32). Nevertheless, as a consequence of the result $\mu \geq\left|s_{0}\right|^{2}$, we have that $\frac{\mu}{\left|s_{0}\right|^{2}} \geq 1$, implying that whenever

$$
\left[\begin{array}{ccccc}
T & T & \ldots & T & T \\
\bullet & Q_{0} & & 0 & 0 \\
\bullet & \bullet & \ddots & 0 & 0 \\
\bullet & \bullet & \bullet & Q_{N} & 0 \\
\bullet & \bullet & \bullet & \bullet & Q_{h}
\end{array}\right]>0
$$

is satisfied, then (32) is also true. Hence, one can use (41) in place of (32) to obtain the results derived from the Propostion 4 


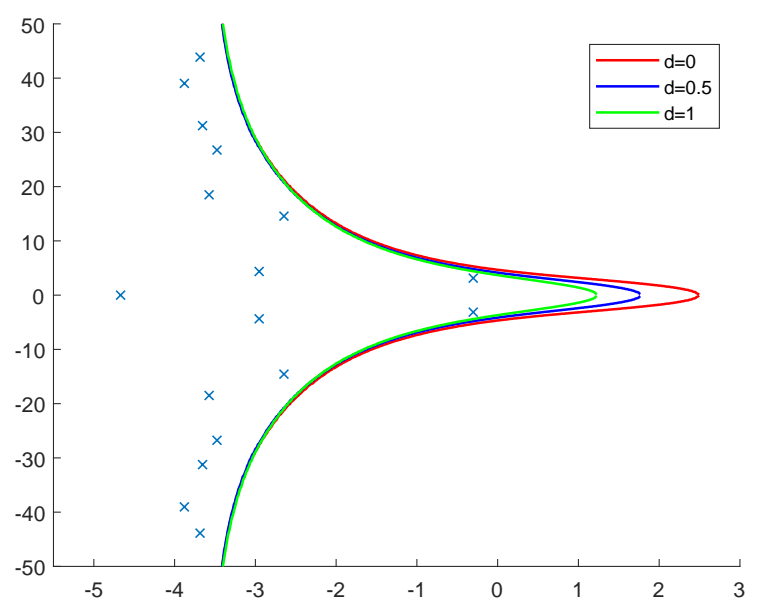

Fig. 4: Envelopes for different values of $d$ - Neutral-type

\section{A. Implementation}

Now, we can make one more time the change of variables $s=z-d$, with $d>0$ and calculate the envelope for z. After this, (30) becomes

$$
\operatorname{det}\left(z I-\tilde{A}_{0}-\sum_{k=1}^{N+1} \tilde{A}_{k} e^{-z \tau_{k}}-z \tilde{H} e^{-z \tau_{h}}\right)=0,
$$

where

$$
\begin{aligned}
\tilde{A}_{0} & =A_{0}+d I, \\
\tilde{A}_{i} & =A_{i} e^{d \tau_{i}}, \forall i \in\{0, \ldots, N\}, \\
\tilde{A}_{N+1} & =-d H e^{d \tau_{h}}, \\
\tilde{H} & =H e^{d \tau_{h}}, \\
\tau_{N+1} & =\tau_{h} .
\end{aligned}
$$

This allows us to perform the same technique used for retarded systems. It remains valid, for the neutral case, the conclusions of subsections II-B and II-C regarding delay independent stability and delay dependent $\alpha$-stability.

Example 3: Consider the matrices

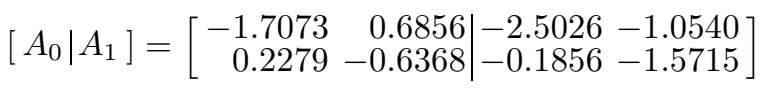

and

$$
[H]=\left[\begin{array}{rr}
0.0558 & 0.0360 \\
0.2747 & -0.1084
\end{array}\right] \text {. }
$$

Figure 4 illustrates the envelopes for $\tau_{1}=\tau_{h}=0.5$.

\section{B. State-feedback for Neutral systems}

We can now adapt the previous result in devise a procedure able to design a state-feedback control law (27) for the linear neutral time-delay system

$$
\dot{x}(t)=\sum_{i=0}^{N} A_{i} x\left(t-\tau_{i}\right)+H \dot{x}\left(t-\tau_{h}\right)+B u(t) .
$$

Theorem 2: Consider the time-delay system (46). If there exist matrices $T=T^{\prime}>0, Q_{i}=Q_{i}^{\prime}>0, \forall i \in\{0, \ldots, N+$

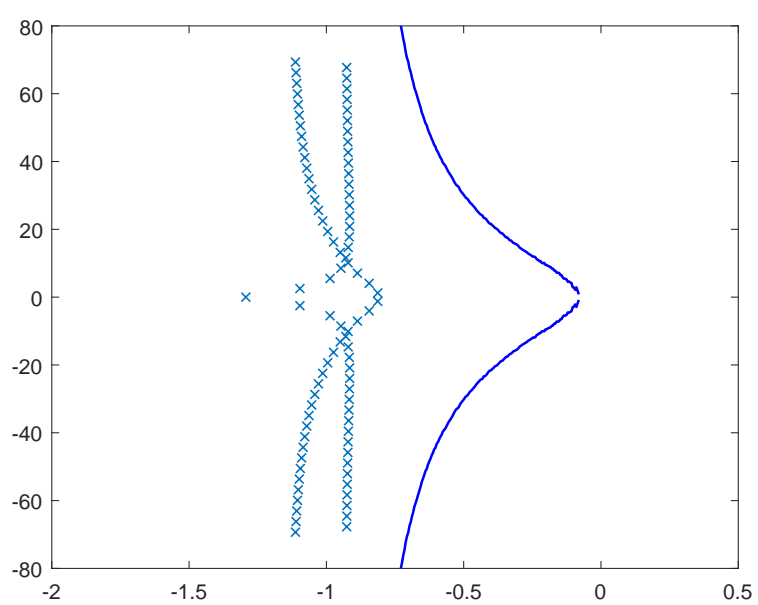

Fig. 5: State-feedback $-\tau_{1}=\tau_{h}=2$

$1\}, Y_{i}, \forall i \in\{0, \ldots, N\}, Q_{h}=Q_{h}^{\prime}>0$ and positive scalars $d$, $\varepsilon$, with $\mu=d^{2}-\varepsilon, \lambda=d$, such that

$$
\left[\begin{array}{ccccc}
\mu T & \left(\tilde{A}_{0} Q_{0}+B_{0} Y_{0}\right) e^{-\lambda \tau_{0}} & \ldots & \tilde{A}_{N+1} Q_{N+1} e^{-\lambda \tau_{h}} & \tilde{H} Q_{h} e^{-\lambda \tau_{h}} \\
\bullet & Q_{0} & \ddots & 0 & 0 \\
\bullet & \bullet & \bullet & 0 & 0 \\
\bullet & \bullet & \bullet & \bullet & \frac{1}{\mu} Q_{h}
\end{array}\right] \geq 0
$$

and (32) are all satisfied, where $\tilde{H}$ and $\tilde{A}_{i}$ for all $i \in$ $\{0, \ldots, N+1\}$ are given by (43) and $B_{i}=B e^{d \tau_{i}}$ for all $i \in\{0, \ldots, N\}$, then the state-feedback controller 27) obtained with the gain matrices $K_{i}=Y_{i} Q_{i}^{-1} \forall i \in\{0, \ldots, N\}$ stabilises the system.

Proof: Applying Schur's complement in (47) with $H \leftarrow \tilde{H}$ and $A_{i} \leftarrow \tilde{A}_{i}+B_{i} K_{i} \forall i \in\{0, \ldots, N\}$, we get

$$
\begin{aligned}
\mu T & \geq \sum_{i=0}^{N} A_{i} Q_{i} A_{i}^{\prime} e^{-2 \lambda \tau_{i}}+\tilde{A}_{N+1} Q_{N+1} \tilde{A}_{N+1}^{\prime} e^{-2 \lambda \tau_{h}} \\
& +\mu H Q_{h} H^{\prime} e^{-2 \lambda \tau_{h}} \\
& \geq \sum_{i=0}^{N} A_{i} Q_{i} A_{i}^{\prime} e^{-2 \lambda \tau_{i}}+\mu H Q_{h} H^{\prime} e^{-2 \lambda \tau_{h}},
\end{aligned}
$$

which is exactly 31 completing the proof.

The stabilisation of neutral delay systems is much more involved than the one of retarded systems due to the possible presence of an infinite number of poles in the right halfplane. We already know from [25] (in the particular case of commensurate delays) that no solution will be provided by Theorem 2 if there is a chain of poles clustering the imaginary axis in the right half-plane.

Example 4: For the matrices (44) and (45), see [21], [22], [23] and [24], the upper bound for the delay was given as 0.8418 . Applying Theorem 2 we designed the following controller $K_{0}=[-37.7924-20.7712], K_{1}=\left[\begin{array}{lll}5.3363 & 3.7375\end{array}\right]$ which guarantees stability for all delays. We illustrate the envelope for $\tau_{1}=\tau_{h}=2$ in Figure 5 , 


\section{CONCLUSIONS}

In this paper we developed a new strategy, based on the use of envelopes, to study stability and to design feedback controllers for linear time-delay systems. We have also provided a new method able to calculate those envelopes through LMIs. Furthermore, this new method is able to cope both with retarded and neutral delay systems. For retarded-type systems, the method was shown to be less conservative than standard results from the literature, such as the eigenvalue approach. For neutral-type systems, the envelope design is entirely original portraying a novel contribution. Additional work is still needed in order to better establish robustness properties of the method and to deal with the output feedback and the filter design problems.

\section{REFERENCES}

[1] W. Michiels, S. I. Niculescu, Stability, Control, and Computation for Time-Delay Systems - an Eigenvalue-Based Approach, SIAM, (2014).

[2] J. P. Richard, Time-delay systems:an overview of some recent advances and open problems, Automatica 39 (2003) 1667-1694.

[3] C. Briat, Stability Analysis of Time-Delay Systems, Springer Berlin Heidelberg, (2015).

[4] J. H. Lee, W. Kim, W. H. Kwon, Memoryless $\mathcal{H}_{\infty}$ Controllers for State Delayed Systems, IEEE Trans. Autom. Contr. 39 (1) (1994) 159-162.

[5] J.-C. Shen, B.-S. Chen, F.-C. Kung, Memoryless Stabilization of Uncertain Dynamic Delay Systems: Riccati Equation Approach, IEEE Trans. Autom. Contr. 36 (5) (1991) 638-640.

[6] E. Fridman, U. Shaked, New Bounded Real Lemma Representations for Time-Delay Systems and Their Applications, IEEE Trans. Autom. Contr. 46 (12) (2001) 1973-1979.

[7] E. Fridman, U. Shaked, A Descriptor System Approach to $\mathcal{H}_{\infty}$ Control of Linear Time-Delay Systems, IEEE Trans. Autom. Contr. 47 (2) (2002) 253-270.

[8] S. I. Niculescu, $\mathcal{H}_{\infty}$ memoryless control with an $\alpha$-stability constraint for time-delay systems: An LMI approach, IEEE Trans. Autom. Control 43 (5) (1998) 739-748.

[9] C. B. Cardeliquio, M. Souza, R. H. Korogui, A. R. Fioravanti, Stability Analysis and State-Feedback Control Design for Time-Delay Systems, European Control Conference (2016) 1686-1690.

[10] T.Mori, H. Kokame, Stability of $\dot{x}(t)=A x(t)+B x(t-\tau)$, IEEE Transactions on Automatic Control 34 (1989) 460-462.

[11] S. S. Wang, Further results on stability of $\dot{x}(t)=A x(t)+B x(t-\tau)$, Systems and Control Letters 19 (1992) 165-168.

[12] R. Bellman, K. Cooke, Differential-difference equations, Academic Press.

[13] G. Strang, Introduction to Linear Algebra, Wellesley-Cambridge Press and SIAM, (2016).

[14] R. Bulirsch, J. Stoer, Introduction to numerical analysis, Springer-Verlag, (1993).

[15] S. Boyd, L. Vandenberghe, Convex Optimization, Cambridge University Press, (2009).

[16] C. A. Desoer, M. Vidyasagar, Feedback Sysrems: Input-Output Propertie, New York: Academic, (1975).

[17] Y. Nesterov, A. Nemirovski, Interior Point Polynomial Methods in Convex Programming: Theory and Applications, SIAM, (1994).

[18] T. Vyhldal, P. Ztek, Mapping Based Algorithm for Large-Scale Computation of Quasi-Polynomial Zeros, IEEE Transactions on Automatic Control 54 (2009) 171-177.

[19] T. Vyhldal, P. Ztek, QPmR v.2 Quasipolynomial rootfinder, algorithm and examples. Advances in Delays and Dynamics, Sringer (2013).

[20] C. Bonnet, A. Fioravanti, J. Partington, Stability of neutral systems with multiple delays and poles asymptotic to the imaginary axis, SIAM 49 (2011) 498-516.

[21] L. X, de Souza CE, Delay-dependent robust stability and stabilization of uncertain linear delay systems: a linear matrix inequality approach, IEEE Transactions on Automatic Control 42 (1997) 1144-1148.

[22] E. Fridman, U. Shaked, An improved stabilization method for linear systems with time-delay, IEEE Transactions on Automatic Control 47 (2002) 1931-1937.
[23] M. Parlaki, Improved robust stability criteria and design of robust stabilizing controller for uncertain linear time-delay systems, International Journal of Robust and Nonlinear Control 16 (2006) 599-636.

[24] J. Sun, G. P. Liu, J. Chen, Delay-dependent stability and stabilization of neutral time-delay systems, International Journal of Robust and Nonlinear Control 19 (2009) 1364-1375.

[25] L. H. V. Nguyen, C. Bonnet, Stabilization of fractional neutral systems with one delay and a chain of poles asymptotic to the imaginary axis, ICFDA, (2014).

[26] W. Kwon, B. Koo, S.M. Lee, Novel Lyapunov-Krasovskii functional with delay-dependent matrix for stability of time-varying delay systems, Applied Mathematics and Computation 320 (2018) 149-157.

[27] B. Zhou, Construction of strict Lyapunov-Krasovskii functionals for time-varying time-delay systems, Automatica 107, (2019) 382-397.

[28] E. Fridman, U. Shaked, Delay-dependent stability and $\mathcal{H}_{\infty}$ control: Constant and time-varying delays, International Journal of Control, 76 (2003) 48-60. 